\title{
Kabuki Syndrome in a Sri Lankan baby
}

\author{
M I Rifaya ${ }^{1}$, S K Rajapakse ${ }^{2}$, T C Liyanage ${ }^{3}$, H H M P P S Herath ${ }^{4}$, RMJC Somaratna ${ }^{4}$
}

Sri Lanka Journal of Child Health, 2009; 38: 36-37

(Key words: Kabuki syndrome, dysmorphic facial features)

\section{Introduction}

Kabuki syndrome (KS), also known as Kabuki makeup syndrome, KMS or Niikawa Kuroki syndrome, is a rare congenital syndrome of unknown aetiology ${ }^{1}$. Diagnosis is based on dysmorphic facial features, skeletal abnormalities, dermatographic abnormalities, developmental delay and mild to moderate mental retardation. It was first described by two Japanese scientists, Niikawa and Kuroki in $1981^{1,2}$. Because of facial resemblance to the make-up of actors in Kabuki, the traditional Japanese theatre, this disorder is referred to as the Kabuki syndrome ${ }^{2,3}$. We report a clinically suspected case of Kabuki syndrome in a Sri Lankan baby.

\section{Case report}

A ten month old male infant, a product of a non consanguineous marriage was born at term, weighing $2.5 \mathrm{~kg}$, following an uncomplicated pregnancy. $\mathrm{He}$ presented with severe persistent vomiting, feeding difficulty and poor weight gain of three months duration which necessitated several hospital admissions. He was initially followed up at the neonatal clinic for hypotonia and facial dysmorphism. He had urinary tract infection at the age of six months. During the hospital stay he developed two episodes of afebrile generalized convulsions. He has a normal elder sibling.

On examination, this hypotonic baby had long palpebral fissures with eversion of outer third, arched eyebrows with sparse outer half, prominent eyelashes, prominent misshapen ears and depressed nasal tip (Figure 1). He also had brachydactyly, clinodactyly, unusually shaped scapulae with high clavicle and hyperextensible joints.

\footnotetext{
${ }^{1}$ Senior Registrar in Paediatrics, ${ }^{2}$ Consultant Paediatrician, ${ }^{3}$ Registrar in Paediatrics, ${ }^{4}$ House Officer in Paediatrics, Teaching Hospital, Kandy.
}

(Received on 20 February 2008. Accepted on 20 March 2008)

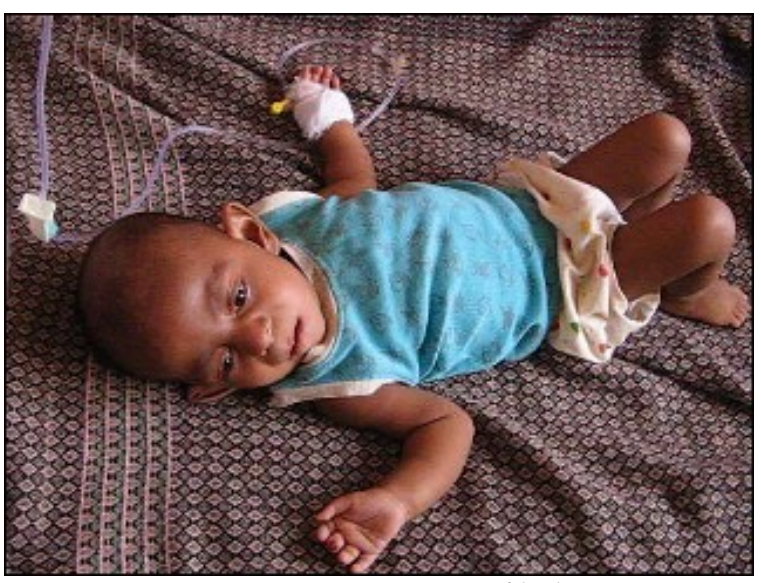

Figure 1 Appearance of baby

His growth parameters were well below the $3^{\text {rd }}$ percentile for his age. The developmental milestones were markedly delayed. No urinary tract or brain abnormalities were detected on ultrasonic examination and the echocardiogram was normal. Barium meal and follow through revealed severe gastro esophageal reflux which was confirmed by 24 hour gastro-esophageal $\mathrm{pH}$ study. Hearing and visual assessments were normal. Electroencephalogram was normal and the chromosomal analysis showed normal karyotype (46XY).

He was initially managed with anti reflux medications and gastric tube feeding with adequate nutrition but there was no significant improvement and the weight gain was very slow. He needed administration of intravenous fluid on several occasions and finally underwent gastric fundoplication following which the frequency of vomiting was reduced. Physical therapy, occupational therapy and speech therapy were arranged and the parents were counselled and regular clinic follow up was made.

During his recent clinic review at one year and four months improvement in the weight gain and developmental milestones were noted but the mother was over concerned about the feeding difficulty even 
though it was explained to her that the hypotonia is a contributing factor to the difficulty with feeding.

\section{Discussion}

The most striking aspects of $\mathrm{KS}$ are the unique facial features which become more obvious with age $\mathrm{ag}^{3,4}$. There is no definitive test for diagnosing KS. Blood studies and chromosomal testing are unhelpful. The diagnosis of $\mathrm{KS}$ is based on the recognition of four (out of five) main characteristics, the distinct facial features being imperative ${ }^{2}$.

- Facial features: long palpebral fissures with eversion of outer third, arched eyebrows with sparse outer half, prominent eyelashes, prominent and/or misshapen ears, and depressed nasal tip.

- Skeletal abnormalities: brachydactyly (shortness of fingers), brachymesophalangy (abnormal shortness of bone), clinodactyly (turning aside) of the fifth finger, and vertebral anomalies including scoliosis.

- Dermatoglyphic abnormalities: including persistent finger fetal pads.

- $\quad$ Developmental delay (mild to moderate).

- Postnatal short stature.

$\mathrm{KS}$ is a complex syndrome with many associated findings such as hypotonia, hyperextensible joints, feeding difficulties, behavioural difficulties, recurrent infections, hearing impairment and/or inner ear malformations, congenital heart defects, renal / urinary tract anomalies, other organ anomalies (less common) - malrotation of colon, anal atresia (closure), diaphragm eventration or hernia, immunological abnormalities and seizures ${ }^{2}$.

Physical therapy, occupational therapy, speech therapy, sensory integration therapy and counselling of parents are the mainstay of management.

\section{Acknowledgements}

We thank Dr. P Jayawardana (Consultant Paediatric Surgeon, TH, Kandy), Dr. Shaman Rajindrajith (Consultant Paediatrician, TH, Ragama), and Dr. Deepthi de Silva (Consultant Clinical Geneticist, TH, Ragama) for their invaluable help.

\section{References}

1. Kabuki syndrome From Wikipedia, the free encyclopedia. Available from: http://en.wikipedia.org/wiki/Kabuki syndrome

2. Facts about Kabuki, Kabuki at a glance C 1998 2008 Kabuki syndrome Net work (KNS). Last updated 16 march 2007.Available from: http://www.kabukisyndrome.com/kabuki.html

3. Kenneth Lyons Jones, M.D editors, Smith's Recognizable Patterns of Human Malformation. 5th ed. Philadelphia W.B. Saunders 1997, p116

4. Adam MP, Hudgins L. Kabuki syndrome: a review. Clinical Genetics 2004: 67: 209-19. 